\title{
The Interaction between Pyrimidine Nucleosides and Benzene in Aqueous Solution, studied by Proton Magnetic Resonance
}

\author{
H.-D. LÜDEMANN \\ Fachbereich Biologie, Lehrstuhl für Physik, Universität Regensburg \\ (Z. Naturforsch. 27 b, 1196-1201 [1972] ; received May 2/June 15, 1972) \\ Interaction, nucleosides, benzene, water, PMR
}

\begin{abstract}
Changes caused in the PMR spectra of aqueous solutions of four pyrimidine nucleosides (uridine, deoxyuridine, cytidine and thymidine) containing benzene $(0.12$ molal $)$ by the variation of concentration and temperature have been investigated. From the data obtained, the participation of the benzene molecules in the stacking of the nucleosides is deduced.
\end{abstract}

Two types of interaction yield the main contributions to the stability of the secondary structure of DNA and RNA molecules in aqueous solution:

1. Specific hydrogen bonding with stringent geometric requirements between the complementary base pairs in the two strands of the double helix of DNA or in helical regions of the RNA chain.

2. Unspecific hydrophobic interactions between the adjacent bases of the nucleosides, with less rigid geometrical requirements.

The second type also occurs in aqueous solutions of the monomeric nucleosides and nucleotides, and is responsible for the solubility enhancement of uncharged polycyclic aromatic compounds in such solutions as compared to pure water ${ }^{1,2}$. The self association has been investigated previously by $\mathrm{PMR}^{3-8}$. The shielding of the ring protons is influenced by the $\pi$-electron-clouds of the adjacent heterocyclic ring structure. A neighbouring aromatic molecule wil cause similar effects and it should be possible to decide via changes in the chemical shifts, whether an aromatic molecule will be found preferably in the vicinity of other nucleosides or if it is distributed at random in the bulk solution. The limited sensitivity of high resolution PMR combined with the low solubility in water of most aromatic compounds containing no polar groups, restricted the investigation of the stacking properties to aromatic ring systems solubilized by an attached polar or even charged group as for instance ethidium bromide ${ }^{9}$. In these cases the attraction between the nucleoside and the aromatic molecule could be caused by electrostatic or dipoledipole interactions as well. However, many of the

Requests for reprints should be sent to Dr. H.-D. LÜDEManN, Lehrstuhl f. Physik, Fachbereich iBologie d. Universität, D-8400 Regensburg, Universitätsstr. 31 . most powerful carcinogenic and mutagenic compounds, for instance 3,4-benzpyren and 20-methylcholanthren containing carbon and hydrogen only but no polar groups, are known to interact strongly with DNA and RNA ${ }^{10-12}$. Benzene, the simplest aromat, is sufficiently soluble in water to allow the observation of proton resonances in a single scan experiment and might serve as a model substance for the less soluble condensed aromatic compounds. Besides, benzene is a dangerous poison itself and its ways of physiological action on the living cell are far from completely understood ${ }^{13}$. In the following experiments it has been investigated whether benzene, dissolved in aqueous solutions of pyrimidine nucleosides, does take part in the association of the bases.

\section{Experimental}

Substances: The nucleosides were obtained from Papierwerke Waldhof-Aschaffenburg AG, Mannheim, BRD, and used without further purification. Heavy water $(99.5 \%$ deuterated) and benzene (Uvasol) were purchased from E. Merck, Darmstadt, BRD, and the hexamethyldisiloxane (HMDS) (puriss.) used for external locking, from Fluka AG., Buchs, Switzerland. The heavy water was saturated with benzene by shaking it with a surplus of benzene in a sealed blask for at least 24 hours. The solutions were prepared immediately before use by mixing weighted amounts of the benzene solution with the appropriate quantities of nucleosides. Concentrations given are molalities. The concentration of the benzene was 0,12 molal $\left(\mathrm{D}_{2} \mathrm{O}\right.$ saturated at $20^{\circ} \mathrm{C}$ ) in all experiments. The concentration of the nucleosides was varied up to the solubility limit. In some cases it was possible to prepare supersaturated solutions that did not yield precipitates during the run of an experiment.

\section{Spectra}

The PMR-spectra were obtained in the CW mode at $100 \mathrm{MHz}$ with a Varian XL-100-15 spectrometer 
equipped with the XL-100 variable temperature accessory in $12 \mathrm{~mm}$ tubings. Frequencies given in this paper are taken against external neat hexamethyldisiloxan (HMDS) contained in a coaxial capillary tubing. They were measured to $\pm 0,1 \mathrm{~Hz}$ with a $\mathrm{U}-4410$ frequency counter. Bulk magnetic susceptibility corrections were not applied. The maximum deviations of the frequencies measured in the same solution in different runs was $\pm 0,4 \mathrm{~Hz}$. This scatter is mainly due to the limited accuracy of the variable temperature accessory, which on frequent checks showed variations up $\pm 2{ }^{\circ} \mathrm{C}$. The spectra were taken between $0^{\circ}$ and $40{ }^{\circ} \mathrm{C}$ in steps of $10^{\circ} \mathrm{C}$.

\section{Results}

The spectra of four pyrimidine nucleosides were obtained: uridine (U) (0.1 to 1.2 molal), deoxyuridine (dU) (0.1 to 0.5 molal), cytidine (C) $(0.1$ to 0.4 molal $)$, and thymidine $(\mathrm{T})$ (0.05 to 0.25

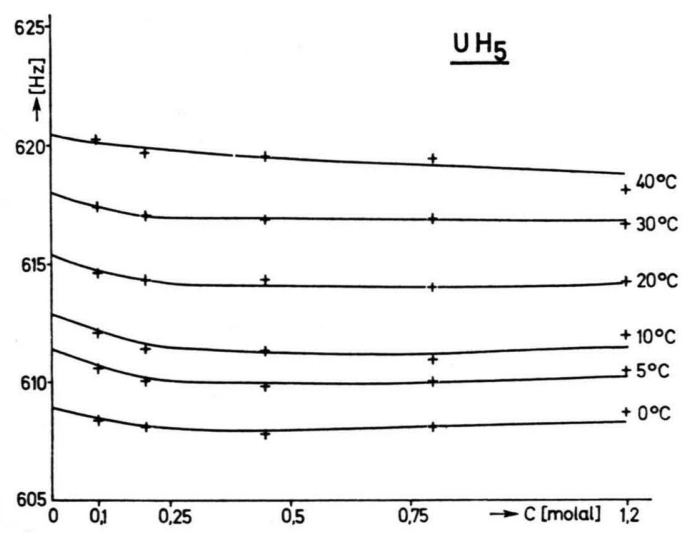

Fig. 1. Concentration and temperature dependence of the chemical shift of the $\mathrm{H}_{5}$-proton resonance in a solution of uridine (U) in deuterium oxide, with 0.12 molal benzene added. (Shifts are taken against HMDS as an external standard.) molal). All resonances in the PMR spectra of these compounds have been assigned to the various protons ${ }^{14}$. In the substances investigated here the signals of the base protons and the proton at the

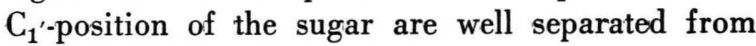
the resonances of the residual protons of the sugar moiety. The latter are crowded around $\tau=4 \mathrm{ppm}$ and their observation in heavy water is often rendered difficult by residual water protons. Figs. 1 to 8 give the positions of the resonance signals of

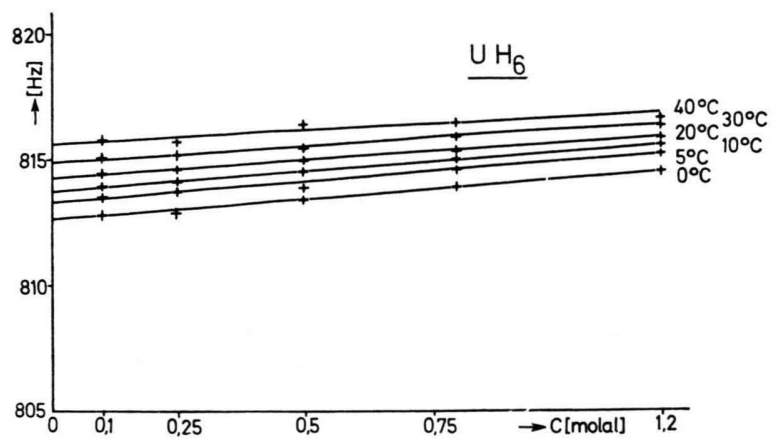

Fig. 3. Concentration and temperature dependence of the chemical shift of the $\mathrm{H}_{6}$-proton resonance in a solution of uridine (U) in deuterium oxide, with 0.12 molal benzene added. (Shifts are taken against HMDS as an external standard.)

the benzene protons, the $\mathrm{H}_{6}$-protons, the $\mathrm{H}_{5}$-protons (in the case of $\mathrm{T}$ : the methyl-protons) and the $\mathrm{H}_{1^{\prime} \text { - }}$ protons of the four nucleosides dissolved in a 0.12 molal solution of benzene in deuterium oxide as a function of concentration at temperatures between $0^{\circ}$ and $40^{\circ} \mathrm{C}$. In addition, solutions with the same concentrations of nucleosides but without benzene were examined. Comparison of the shifts observed at equal temperatures and concentrations in these
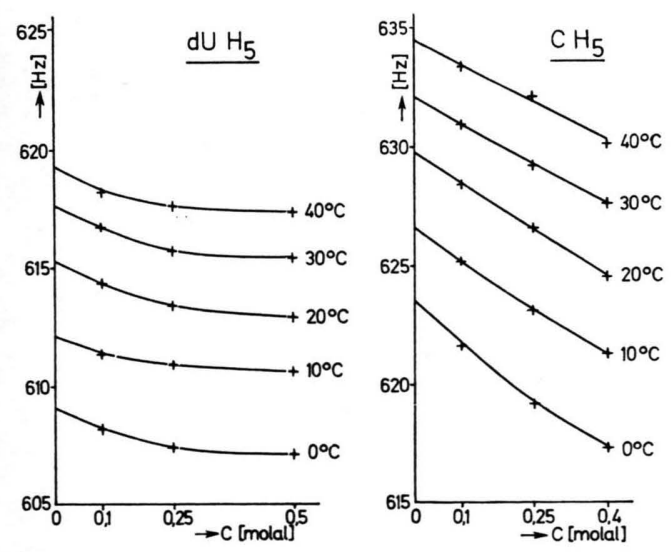

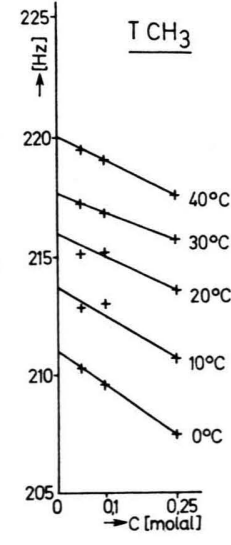

Fig. 2. Concentration and temperature dependence of the chemical shift of the $\mathrm{H}_{5}$-proton (in the case of thymidine $\mathrm{CH}_{3}$-protons) in solutions of deoxyuridine (dU), cytidine (C) and thymidine (T) in deuterium oxide, with 0.12 molal benzene added. (Shifts are taken against HMDS as an external standard.) 

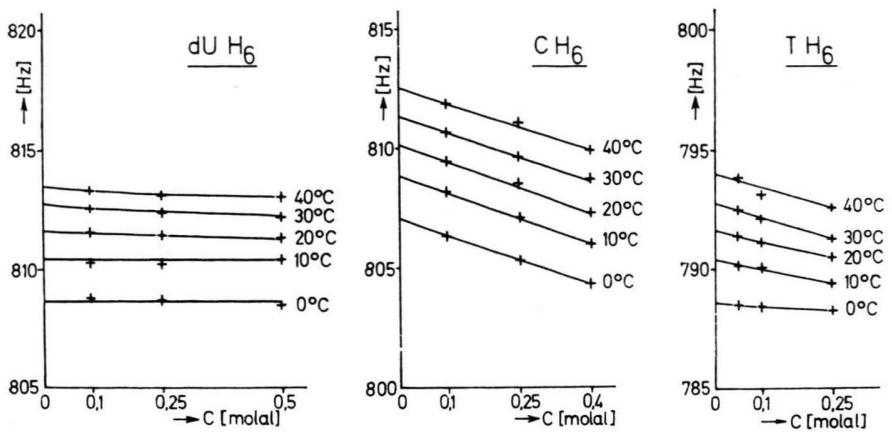

solutions to those containing benzene showed, that the resonances of the base protons and the $\mathrm{H}_{1^{\prime}}$. protons are displaced to higher fields by 0.2 to $0.8 \mathrm{~Hz}$ on the addition of benzene. These differences are rising with decreasing temperature and concentration. The observed effects are beyond the experimental uncertainties, but considering the reproducibility of $\pm 0.4 \mathrm{~Hz}$ a detailed discussion is not attempted.

\section{Discussion}

Fig. 5. Concentration and temperature dependence of the chemical shift of the $\mathrm{H}_{1^{\prime}}$-proton resonance in a solution of uridine (U) in deuterium oxide, with 0.12 molal benzene added. (Shifts are taken against HMDS as an external standard.)
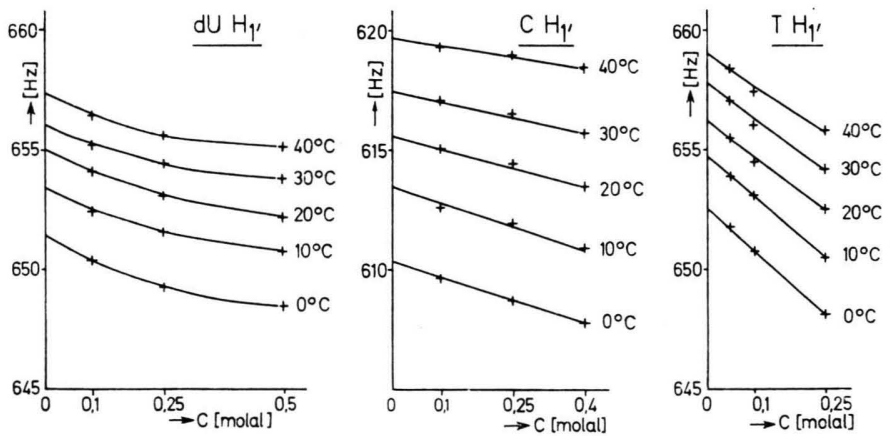

Fig. 6. Concentration and temperature dependence of the chemical shift of the $\mathrm{H}_{1^{\prime}}$-proton in solutions of deoxyuridine (dU), cytidine $(\mathrm{C})$ and thymidine (T) in deuterium oxide, with 0.12 molal benzene added. (Shifts are taken against HMDS as an external standard.)

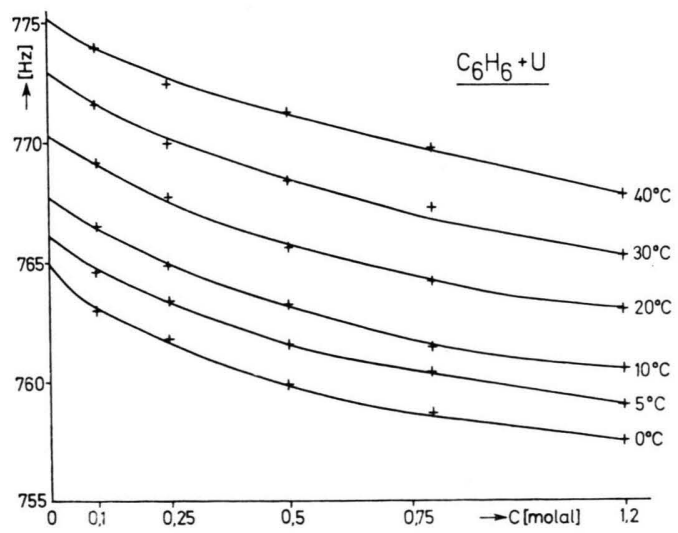

fects caused by the $\pi$-electron-clouds of neighbouring molecules arranged in face to face stacks ${ }^{15-17}$. The magnitude and geometric conditions of this effect have been calculated by JoHNSON and BoveY ${ }^{15}$. Lately this calculations have been extended by Giessner-Prettre, and Pullman ${ }^{18}$ to some naturally occuring pyrimidine and purine bases. The latter calculations contrary to earlier statements ${ }^{19}$

Fig. 7. Concentration and temperature dependence of the chemical shift of the benzene-protons resonance in a solution of uridine (U) in deuterium oxide with 0.12 molal benzene added. (Shifts are taken against HMDS as an external stardard.) 

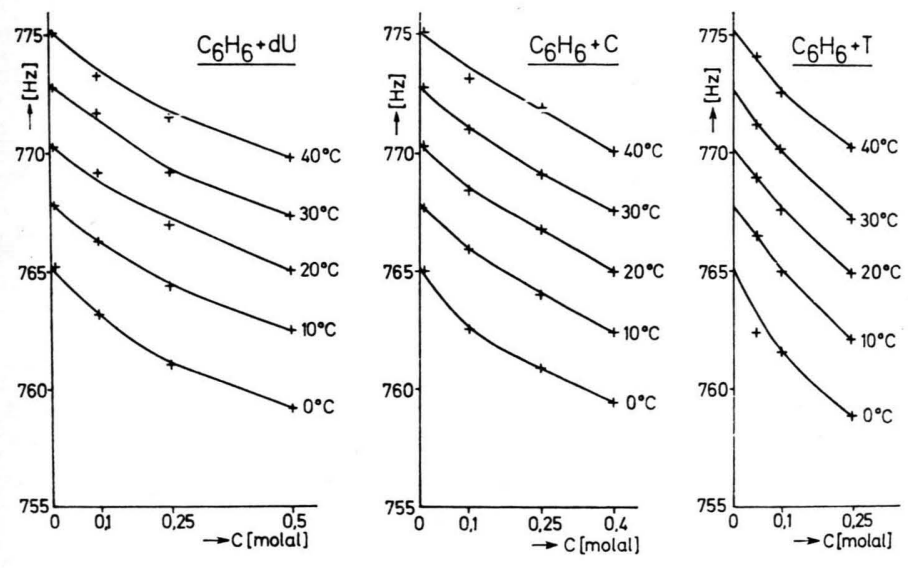

Fig. 8. Concentration and temperature dependence of the chemical shift of the benzene-protons in solutions of deoxyuridine (dU), cytidine (C) and thy. midine $(\mathrm{T})$ in deuterium oxide with 0.12 molal benzene added. (Shifts are taken against HMDS as an external standard.)

reveal that the difference of shifts expected for a completely stacked monomer in solution compared to the isolated molecule in the case of the pyrimidine bases is almost one order of magnitude smaller than for benzene. A comparison of the concentration and temperature dependence of the shifts, obtained for protons at the same position in different nucleosides, shows the following similarities: Changes of the chemical shift of the $\mathrm{H}_{6}$-proton are always smaller than changes of the $\mathrm{H}_{5}$-position. The concentration dependence of the $\mathrm{H}_{1^{\prime}}$-proton shift is higher in the deoxyribosides than in the ribosides, while for the temperature dependence the reverse is true.

\section{Concentration dependence}

The association of the bases in stacks has been measured by osmometric methods ${ }^{20,21}$. According to these results the asociation increases in the sequence $\mathrm{U}<\mathrm{C}<\mathrm{T}$. The concentration dependence of the chemical shift of the base protons (Fig. 1, $2 \mathrm{H}_{6}$ and Fig. $3,4 \mathrm{H}_{5}$, respectively, $-\mathrm{CH}_{3}$ in $\mathrm{T}$ ) has the sequence $\mathrm{U}<\mathrm{T}<\mathrm{C}$. Only the benzene protons (Figs. 7, 8) are shielded with increasing concentration in the sequence suggested by the osmometric data for self-association. Crystallographic data show ${ }^{22}$ that the $\mathrm{H}_{6}$-proton of the pyrimidines in the anti conformation with respect to the glycosidic bond, is very close to the $\mathrm{O}_{5^{\prime}}$ of the sugar moiety. An intramolecular hydrogen bond connecting $\mathrm{C}_{6}$ via the $\mathrm{H}_{6}$ to $\mathrm{O}_{5^{\prime}}$ is suggested for this geometry. It is reasonable to assume a similar sterical arrangement in the dissolved molecules ${ }^{23}$ especially under consideration of the recent PMR results obtained by BARRY et al. ${ }^{24}$ who used lanthanide shift reagents for an analysis of the pre- ferred conformation of mononucleotides in solution. The anti conformation, with, or without a hydrogen bond, would inhibit the approach of any other molecule, solvent or nucleoside, to the vicinity of $\mathrm{H}_{6}$ compared to a proton or methyl group in the 5-position of the pyrimidine bases, the latter being freely exposed to the surrounding medium. This assumption may be supported by comparison of the $\mathrm{H}_{6}$ shifts observed in thymidine and cytidine. Though the first is known to associate stronger ${ }^{25}$ than the second, the concentration dependence of the shifts is significantly greater in cytidine than in thymidine, since presumably the bulkier methyl group of the thymidine is a more effective obstacle to approaching molecules than the $\mathrm{H}_{5}$-proton of cytidine. The shifts of the uridine protons (Figs. 13,5 ) show the smallest concentration dependence. The curves obtained for $\mathrm{H}_{6}$ (Fig. 1) seem to pass through a shallow minimum while the $\mathrm{H}_{5}$ (Fig. 3) resonance is even shifted to lower fields with increasing concentration. Both observations do not contradict the statements given above.

The ring currents in uracil according to the calculations of Giessner-Prettre and Pullman ${ }^{18}$ are weaker than in cytosine. Consequently, the influence of the ring currents on the shifts in aqueous uridine solutions can easily be masked by minor changes in the bulk magnetic susceptibility.

A similar purely sterical explanation might be given for the different concentration dependence of the $\mathrm{H}_{1^{\prime}}$-proton shift in the ribosides and deoxyribosides (Figs. 5, 6). Here the replacement of the hydroxyl-group at the 2 '-position by a hydrogen exposes the $\mathrm{H}_{1^{\prime}}$ more freely to the surrounding medium. 
Shift of the benzene protons: Even minor changes in the structure of the nucleosides investigated here, show up in differences in the chemical shift of the benzene protons (Figs. 7, 8, 9). This proves,

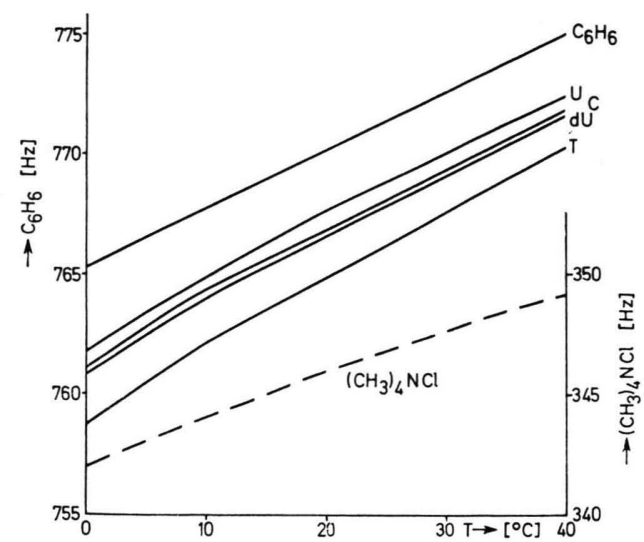

Fig. 9. Shift of the benzene-protons as a function of temperature in a 0.12 molal solution in deuterium oxide $\left(\mathrm{C}_{6} \mathrm{H}_{6}\right)$ and with 0.25 molal uridine (U), deoxyuridine (dU), cytidine (C) and thymidine (T) added (left scale), and temperature dependence of the methyl-protons of a tetramethylammoniumchloride solution $(c=1.0 \%)$ in deuterium oxide (right scale).

(Shifts are taken against HMDS as an external standard.)

together with the fact, that no similar effects were found for solutions of equal concentration in dimethylsulfoxide, the participation of benzene in the association equilibria of the mononucleosides. Benzene however, can produce shielding effects an order of magnitude higher than those caused by the face to face approach of the pyrimidine bases. Still, the influence of the ring currents in the nucleosides on the benzene protons at comparable concentrations is greater than the effect of benzene on the base protons. Two likely explanations might be given for this observation; 1) In the case of face to face stacking between benzene and the nucleosides the center of the benzene ring is preferably found above or below the nitrogen atoms or carbonyl groups of the pyrimidine rings. The arrangement would bring some benzene protons into the regions of the highest diamagnetic shift of the pyrimidine bases while leaving the $\mathrm{H}_{5}$ and $\mathrm{H}_{5}$ protons of the bases rather unaffected by shielding from the benzene.

2) if attachment of the benzene rings perpendicular to the pyrimidine bases occurs the benzene ring centering on one of the carbonyl groups would form a kind of a charge-transfer-complex. For both ways of interaction the influence on the ben- zene and base protons will be similar. No decision can be made between these two possibilities on the basis of proton shift measurements alone.

\section{Effects of temperature}

Decreasing temperature results in a high field shift of all proton resonances. At first sight, one is tempted to explain this increase of shielding in the same way as the concentration effects. If, however, the shifts were caused by association of the bases, the difference between two signals obtained at different concentrations should become smaller with decreasing concentration and at infinite dilution all isotherms should join.

Since obviously the isotherms in the Figs. 1 to 8 with small but significant deviations run parallel, the assumption given above does not hold. In some of their investigations KreISHMAN and CHAN ${ }^{26}$ used tetramethylammoniumchloride as an water soluble "inert" reference substance. In a preliminary experiment during this work the chemical shift of the tetramethylammoniumchloride protons in a $1 \%$ aqueous solution was measured against an external lock capillary filled with HMDS. Cooling the solution in $10^{\circ} \mathrm{C}$ steps from $40{ }^{\circ} \mathrm{C}$ to $0^{\circ} \mathrm{C}$ gave a high field shift of $7.0 \mathrm{~Hz}$. Calculations of the change of the differences between the magnetic volume susceptibilities with temperature under the most unfavorable assumptions for the coefficients of thermal expansion yielded a maximum shift of $2.5 \mathrm{~Hz}$. In Fig. 9 the shifts of the benzene protons (concentration 0.12 molal) in pure heavy water and in aqueous 0.25 molal solutions of $\mathrm{U}, \mathrm{dU}, \mathrm{C}$ and $\mathrm{T}$ are plotted as functions of temperature. In the lower part of the figure the resonance of the methyl protons of a $1 \%$ tetramethylammoniumchloride aqueous solution is plotted. This compound, completely ionized in water, can certainly neither associate nor produce any ring current effects. The explanation should consequently be searched for in the surrounding hydration sphere. A decrease in temperature will allow the water molecules surrounding the hydrophobic parts of a dissolved molecule to arrange more regularly and this may lead to a slightly higher electron density at the proton. This qualitative explanation is further corroborated by comparing the change in chemical shift with temperature among the different protons observed here. The $\mathrm{H}_{5}$, $\mathrm{H}_{1^{\prime}}$ and the benzene protons situated on positions in their molecules exposed to the surrounding water 
are shifted between $40{ }^{\circ} \mathrm{C}$ and $0{ }^{\circ} \mathrm{C}$ approximately $10 \mathrm{~Hz}$ upfield, while the $\mathrm{H}_{6}$-protons, which in the anti conformation of the nucleosides are hidden in a recess of the molecule, can be influenced much less by the solvent. Accordingly the shifts due to a temperature change of $40^{\circ} \mathrm{C}$ vary by 3 to $5 \mathrm{~Hz}$ only.

From the data presented here, one can deduce that benzene dissolved in aqueous solutions of pyrimidine nucleosides does take part in the stacking or association of the bases. It does not seem sensible to attempt the determination of the thermodynamic parameters governing the association process from the shifts observed, mainly because the effects observed are on the limit of detectability and because

1 P. O. P. Ts'o and P. Lu, Proc. Nat. Acad. Sci. USA 51, 17 [1964].

2 E. Boyland and G. Green, Brit. J. Cancer 16, 507 [1962].

3 O. JaRdetzky and C. D. Jardetzky, J. Amer. chem. Soc. 82, 222 [1960].

4 O. Jardetzky, P. Pappas, and N. G. Wade, J. Amer. chem. Soc. 85, 1657 [1963].

5 F. B. Howarrd, J. Frazier, M. F. Singer, and H. T. Mrles, J. molecular Biol. 16, 415 [1966].

6 P. O. P. Ts'o, M. P. Schweizer, and O. P. Hollis, Ann. New York Acad. Sci. 158, 256 [1969].

7 W. H. HuANG and P. O. P. Ts'o, J. molecular Biol. 16, 523 [1966].

8 L. KATZ, J. molecular Biol. 44, 279 [1966].

9 G. P. Kreishman, S. J. Chan, and W. Bauer, J. molecular Biol. 61, 45 [1971].

10 J. K. Ball, J. A. McCarter, and M. E. Smith,Biochim. biophysica Acta [Amsterdam] 103, 275 [1965].

11 E. De Maeyer, J. De Maeyer, and Guiguard, Science [Washington] 146, 650 [1964].

12 S. N. Cohen and K. L. Yielding, J. biol. Chemistry 240, 3123 [1965].

13 D. W. PARKe and R. T. Williams, Biochem. J. 46, 236 [1950]. of the impossibility to ascribe them quantitatively to definite structures and phenomena. The investigation of the variation of the spin-lattice relaxation times of the different nucleoside protons and the benzene protons as functions of concentration and temperature, by application of the Fourier-transform-nuclear magnetic resonance and the observation of nuclei other than hydrogen will probably yield more detailed insight into the stacking interaction among nucleosides and aromatic hydrocarbons, such investigations are under way in this laboratory.

It is a pleasure to thank Dr. E. von Goldammer and Prof. Dr. Adolf Müller-Broich for many helpful discussions.

14 H. A. SzYmANSKI and R. E. Yelin, NMR Band Handbook, IFI/Plenum N.Y., Washington 1968.

15 C. E. Johnson and F. A. BoveY, J. chem. Physics 29, 1012 [1959].

16 J. S. Waugh and R. W. Fessenden, J. Amer. chem. Soc. 79, 846 [1957].

17 P. Saszlo, Progress in NMR Spectroscopy, 3, p. 231, ed. by J. W. Emsley, J. Feeney, L. H. Sutcliffe, Pergamon Press, Oxford 1967.

18 C. Giessner-Prettre and B. Pullman, J. Theor. Biol. 27. 87 [1970].

19 O. JARDETZKy, Biopolymers Symposia 1, 501 [1964].

20 P. O. P. Ts'o, J. S. Melvin, and A. C. Olson, J. Amer. chem. Soc. 85, 1289 [1962].

21 A. D. Broom, M. P. Schweizer, and P. O. P. Ts'o, J. Amer. chem. Soc. 89, 3612 [1967].

22 N. C. Seeman, J. L. Sussman, H. M. Berman, and S. H. KIM, Nature New Biology 233, 90 [1971].

23 B. J. Blackburn, A. A. Grey, J. C. P. Smith, and F. E. Hruska, Canad. J. Chem. 48, 2866 [1970].

24 C. D. Barry, A. C. T. North, J. A. Glasel, R. J. P. WilLiams, and A. V. XAvier, Nature [London] 232, 5308 [1971].

25 P. O. P. Ts'o, Ann. New York Acad. Sci. 153, 785 [1969].

26 G. P. Kreishman and S. J. Chan, Biopolymers 10, 159 [1971]. 